\title{
Mycophenolate mofetil plus prednisone for inducing remission of Henoch-Schönlein purpura nephritis: a retrospective study ${ }^{*}$
}

\author{
Fei HAN, Liang-liang CHEN, Ping-ping REN, Jing-yun LE, Pei-jing CHOONG, \\ Hong-ju WANG, Ying XU, Jiang-hua $\mathrm{CHEN}^{\dagger \dagger}$ \\ (Kidney Disease Center, the First Affiliated Hospital, School of Medicine, Zhejiang University 1 \\ Key Laboratory of Kidney Disease Prevention and Control Technology, Zhejiang Province I \\ the Third Grade Laboratory under the National State, Administration of Traditional Chinese Medicine, Hangzhou 310003, China) \\ †E-mail: chenjianghua@zju.edu.cn \\ Received Dec. 7, 2014; Revision accepted Mar. 5, 2015; Crosschecked Aug. 10, 2015
}

\begin{abstract}
Objective: The treatment of Henoch-Schönlein purpura (HSP) with moderate proteinuria remains controversial. We retrospectively analyzed the efficacy of immune suppressants, with a particular emphasis on mycophenolate mofetil (MMF). Methods: Ninety-five HSP patients with moderate proteinuria (1.0-3.5 g/24 h) after at least three months of therapy with angiotensin-converting enzyme inhibitor (ACEI) or angiotensin receptor blocker (ARB) were divided into three groups: an MMF group $(n=33)$ that received MMF $1.0-1.5 \mathrm{~g} / \mathrm{d}$ combined with prednisone $(0.4-0.5 \mathrm{mg} /(\mathrm{kg} \cdot \mathrm{d}))$, a corticosteroid (CS) group $(n=31)$ that received full-dose prednisone $(0.8-1.0 \mathrm{mg} /(\mathrm{kg} \cdot \mathrm{d}))$, and a control group ( $n=31)$. Patients in the MMF and CS groups continued to take ACEI or ARB at the original dose. The patients in the control group continued to take ACEI or ARB but the dose was increased by $(1.73 \pm 0.58)$-fold. The patients were followed up for 6-78 months (median 28 months). Results: The baseline proteinuria was higher in the MMF group $((2.1 \pm 0.9) \mathrm{g} / 24 \mathrm{~h})$ than in the control group $((1.6 \pm 0.8) \mathrm{g} / 24 \mathrm{~h})(P=0.039)$. The proteinuria decreased significantly in all groups during follow-up, but only in the MMF group did it decrease significantly after the first month. At the end of follow-up, the proteinuria was $(0.4 \pm 0.7) \mathrm{g} / 24 \mathrm{~h}$ in the MMF group and $(0.4 \pm 0.4) \mathrm{g} / 24 \mathrm{~h}$ in the CS group, significantly lower than that in the control group $((0.9 \pm 1.1) \mathrm{g} / 24 \mathrm{~h})$. The remission rates in the MMF group, CS group, and control group were respectively $72.7 \%, 71.0 \%$, and $48.4 \%$ at six months and $72.7 \%, 64.5 \%$, and $45.2 \%$ at the end of follow-up. The overall number of reported adverse events was 17 in the MMF group, 30 in the CS group, and 6 in the control group $(P<0.001)$. Conclusions: MMF with low-dose prednisone may be as effective as full-dose prednisone and tend to have fewer adverse events. Therefore, it is probably superior to conservative treatments of adult HSP patients with moderate proteinuria.
\end{abstract}

Key words: Henoch-Schönlein purpura, Nephritis, Mycophenolate mofetil, Remission doi: 10.1631 jzus.B1400335

Document code: A

CLC number: R692.3

\section{Introduction}

Henoch-Schönlein purpura (HSP) is a leukocytoclastic vasculitis of small vessels associated with

\footnotetext{
${ }^{\ddagger}$ Corresponding author

* Project supported by the National Key Technology R \& D Program of China (No. 2013BAI09B04) and the Medical Research Funds from the Bureau of Health of Zhejiang Province (No. 2013KYA072), China (D) ORCID: Fei HAN, http://orcid.org/0000-0002-7089-6223; Jianghua CHEN, http://orcid.org/0000-0002-3282-3998

(C) Zhejiang University and Springer-Verlag Berlin Heidelberg 2015
}

the deposit of immunoglobulin A (IgA) immune complex. It is characterized by palpable purpura, which is the mandatory criterion, and presentations commonly include abdominal pain, arthritis, and nephritis (Ozen et al., 2006). Henoch-Schönlein purpura nephritis (HSPN) was reported in $30 \%-80 \%$ of HSP patients and may result in chronic renal failure in up to $11 \%-38 \%$ of patients in long-term follow-up (Pillebout et al., 2002; Ronkainen et al., 2002; Kellerman, 2006; Shenoy et al., 2007). A high level of proteinuria was associated with a poor renal prognosis 
(Coppo et al., 1997; 2006; Pillebout et al., 2002). After a trial of angiotensin-converting enzyme inhibitor (ACEI) and angiotensin receptor blocker (ARB), the KDIGO (Kidney Disease: Improving Global Outcomes) Glomerulonephritis Work Group (2012) suggested HSPN patients with persistent proteinuria greater than $1 \mathrm{~g} / 24 \mathrm{~h}$ be treated with corticosteroid (CS). However, limited evidence was available about the use of immune suppressants such as CS in HSPN patients, especially in adults. A randomized, placebocontrolled trial revealed that renal symptoms resolved in $61 \%$ of HSP patients after prednisone treatment, compared with $34 \%$ of placebo patients (Ronkainen et al., 2006). However, this trial provided data on outcomes only at 6 months.

Immunosuppressive agents have been used in the treatment of patients with severe HSPN (Foster et al., 2000; Kawasaki et al., 2004). One recent prospective trial compared the effects of prednisone with or without cyclophosphamide (CYC) in severe HSP patients after 12 months and revealed that adding CYC provided no extra benefits (Pillebout et al., 2010). Mycophenolic acid, the active metabolite of mycophenolate mofetil (MMF), is now used in the treatment of systemic lupus erythematosus (SLE), antineutrophil cytoplasmic autoantibody (ANCA)associated systemic vasculitides, and IgA nephropathy (IgAN) (Chan et al., 2000; Ginzler et al., 2005; Hu et al., 2008; Tang et al., 2010; Han et al., 2011). We compared the effects of MMF and CYC in patients with microscopic polyangiitis and found that the remission rates in both groups were similar, whereas there was a higher proportion of patients with a serum creatinine less than $133 \mu \mathrm{mol} / \mathrm{L}$ in the MMF group $(63.2 \%)$ than in the $\mathrm{CYC}$ group $(31.8 \%)$ at 6 months (Han et al., 2011).

In this study, we retrospectively divided adult HSPN patients with moderate proteinuria (greater than $1.0 \mathrm{~g} / 24 \mathrm{~h}$ and less than $3.5 \mathrm{~g} / 24 \mathrm{~h}$ ) after a therapy of at least three months of ACEI or ARB into three groups: in one group, the patients continued to take ACEI or ARB but the dose was increased; in the other two groups, we added either full-dose prednisone or MMF combined with low-dose prednisone and maintained ACEI or ARB at the original dose. The effects on remission and the frequency of adverse events were compared among these groups.

\section{Materials and methods}

The study protocols conformed to the provisions of the Declaration of Helsinki and were approved by the Ethic Committee of our hospital. We retrospectively analyzed all the HSPN patients that received renal biopsy and were followed up in the Kidney Disease Center of the First Affiliated Hospital, School of Medicine, Zhejiang University (Hangzhou, China) between Jan. 2007 and June 2013. The diagnosis of HSP was in accordance with the criteria defined by Ozen et al. (2006). The deadline for follow-up was Dec. 31, 2013. Patients meeting the following criteria were included in the analysis: (1) proteinuria greater than $1.0 \mathrm{~g} / 24 \mathrm{~h}$ or less than $3.5 \mathrm{~g} / 24 \mathrm{~h}$ after at least three months of therapy with ACEI or ARB; (2) an estimated glomerular filtration rate (eGFR; calculated by the simplified (modification of diet in renal disease) MDRD equation) higher than $60 \mathrm{ml} / \mathrm{min}$ per $1.73 \mathrm{~m}^{2}$; (3) a follow-up period of at least 6 months. These patients were divided into three groups (MMF group, CS group, and control group) according to the therapy added after at least three months of treatment with ACEI or ARB. For patients in the MMF group, we added oral MMF $1.0 \mathrm{~g} / \mathrm{d}(1.5 \mathrm{~g} / \mathrm{d}$ for patients with a body weight of $\geq 70 \mathrm{~kg}$ ) combined with prednisone $(0.4-0.5 \mathrm{mg} /(\mathrm{kg} \cdot \mathrm{d}))$; for those in the CS group, we added prednisone $(0.8-1.0 \mathrm{mg} /(\mathrm{kg} \cdot \mathrm{d}))$. Patients in both the MMF and CS groups continued to take ACEI or ARB at the original dose. The patients in the control group continued to take ACEI or ARB but the dose was increased. In the MMF group, we deducted $5 \mathrm{mg}$ of prednisone every 2 weeks after $6-8$ weeks, and maintained $10-15 \mathrm{mg}$ for 3-6 months before gradually tapering to a stop; the dose of MMF was maintained for at least 6 months, and then was gradually reduced; the duration of MMF plus CS therapy was one year. In the CS group, we deducted $5 \mathrm{mg}$ prednisone every week after $6-8$ weeks till the dose was halved, then deducted $5 \mathrm{mg}$ every 2 weeks, and then maintained $10-15 \mathrm{mg}$ for 3-6 months before gradually tapering to a stop; the duration of CS therapy was one year.

Renal pathological changes were independently classified according to the International Study of Kidney Diseases in Children (ISKDC) classification (Counahan et al., 1977). Pathological changes, such as global glomerular sclerosis, crescent formation, 
glomerular segmental necrosis, mesangial proliferation, vasculitis, and interstitial inflammation, were analyzed. The interstitial infiltrates and tubular atrophy were graded according to the distribution area: mild, less than $25 \%$; moderate, greater than $25 \%$, but less than $50 \%$; and severe, greater than $50 \%$ of the total area.

All the patients were followed up each month during the first year and every three months subsequently. In each follow-up visit, the patients underwent physical examination and laboratory screening, including blood cell counts, renal and liver function tests, blood glucose and lipid levels, urine tests, C-reactive protein (CRP) level, and IgA level. The results were evaluated at 1,3 , and 6 months and at the end of follow-up. Remission was defined as proteinuria $\leq 0.3 \mathrm{~g} / 24 \mathrm{~h}$, without an obvious decrease in the eGFR level. For adverse effects, hyperlipidemia was defined as a blood cholesterol level greater than $5.17 \mathrm{mmol} / \mathrm{L}$ and (or) triglycerides greater than $2.3 \mathrm{mmol} / \mathrm{L}$; hyperglycemia was defined as a fasting plasma glucose concentration greater than $6.1 \mathrm{mmol} / \mathrm{L}$; hypertension was defined as systolic blood pressure greater than $140 \mathrm{mmHg}$ and (or) diastolic blood pressure greater than $90 \mathrm{mmHg}$; alanine aminotransferase elevation was defined as a serum alanine aminotransferase concentration greater than $40 \mathrm{U} / \mathrm{L}$.

The analysis was performed using SPSS 16.0 software (SPSS Inc., Chicago, IL, USA). Numerical data were expressed as mean \pm standard deviation (SD) and comparisons were made within groups between each follow-up using a one-way analysis of variance (ANOVA) test, and among groups using the $t$-test. Categorical data were expressed as counts or percentages and compared using a $\chi^{2}$ test. The MannWhitney test was used for nonparametric comparisons. $P<0.05$ was considered significant.

\section{Results}

A total of $205 \mathrm{HSPN}$ patients were screened and 95 who met the criteria were included in the analysis. There were 33 patients in the MMF group, 31 in the CS group, and 31 in the control group. The baseline clinical and pathological characteristics of the three groups are shown in Tables 1 and 2, respectively. At baseline, the proteinuria level was higher in the MMF group $((2.1 \pm 0.9) \mathrm{g} / 24 \mathrm{~h})$ than that in the control group ((1.6 \pm 0.8$) \mathrm{g} / 24 \mathrm{~h}, P=0.039)$. There were no significant differences in serum albumin or eGFR levels among the three groups. The patients had type II or type III HSPN renal pathology. There were no significant differences in the severity of pathological changes such as glomerular sclerosis, crescent formation, mesangial proliferation, segmental necrosis, or interstitial infiltrates among the three groups.

In the control group, the dose of ACEI or ARB was increased by $(1.73 \pm 0.58)$-fold (range 1.0-3.0fold), and the mean arterial pressure (MAP) level of the patients after the first month was lower than baseline $((96.9 \pm 10.3) \mathrm{mmHg}$ vs. $(105.5 \pm 9.7) \mathrm{mmHg}$, $P=0.008$ ), whereas at the other follow-up months there were no significant differences in MAP level compared with baseline (Fig. 1). In the MMF group, the dose of prednisone was $(25.3 \pm 5.0) \mathrm{mg} / \mathrm{d}$ and the dose of MMF was $(1.1 \pm 0.2) \mathrm{g} / \mathrm{d}$ at baseline; at the end of follow-up (median 28 months), 28 patients (84.8\%) had stopped prednisone and among these patients, 21 (63.6\%) had also stopped MMF. In the CS group, the dose of prednisone was $(55.7 \pm 7.4) \mathrm{mg} / \mathrm{d}$ at baseline; at the end of follow-up (median 29 months), 16 patients $(51.6 \%)$ had stopped prednisone.

The follow-up data from the three groups are shown in Fig. 1. The proteinuria level decreased significantly and the serum albumin level increased significantly during follow-up within all groups. After the first month, in the MMF group the proteinuria level decreased compared with baseline $((1.3 \pm 1.2) \mathrm{g} /$ $24 \mathrm{~h}$ vs. $(2.1 \pm 0.9) \mathrm{g} / 24 \mathrm{~h}, P=0.005)$ and the serum albumin level increased compared with baseline $((42.1 \pm 6.7) \mathrm{g} / \mathrm{L}$ vs. $(38.6 \pm 5.3) \mathrm{g} / \mathrm{L}, P=0.023)$, whereas there were no significant differences in the proteinuria or serum albumin levels after the first month in the CS or control group compared with baseline. At the end of follow-up, the proteinuria level was $(0.4 \pm 0.7) \mathrm{g} / 24 \mathrm{~h}$ in the MMF group and $(0.4 \pm 0.4) \mathrm{g} /$ $24 \mathrm{~h}$ in the CS group; both were significantly lower than the level in the control group $((0.9 \pm 1.1) \mathrm{g} / 24 \mathrm{~h}$, $P<0.05$ ). There were no significant changes in serum albumin level among the three groups at each followup time. The eGFR kept stable within the normal range in all groups during follow-up.

The remission rates in the MMF, CS, and control groups were respectively $72.7 \%, 71.0 \%$, and $48.4 \%$ 
Table 1 Baseline clinical characteristics of the patients in the MMF, CS, and control groups

\begin{tabular}{|c|c|c|c|c|}
\hline \multirow{2}{*}{ Characteristics } & \multicolumn{3}{|c|}{ Group } & \multirow{2}{*}{$P$-value } \\
\hline & MMF & $\mathrm{CS}$ & Control & \\
\hline Cases, $n$ & 33 & 31 & 31 & \\
\hline Male/female, $n$ & $13 / 20$ & $14 / 17$ & $12 / 19$ & 0.348 \\
\hline Age (year) & $31 \pm 15$ & $29 \pm 16$ & $35 \pm 15$ & 0.328 \\
\hline Follow-up time (month), median (range) & $28(6-78)$ & $29(6-63)$ & $26(6-65)$ & 0.623 \\
\hline Disease duration before inclusion (month), median (range) & $4.5(3-360)$ & $5.0(4-96)$ & $4.0(3-60)$ & 0.215 \\
\hline \multicolumn{5}{|l|}{ Extra renal manifestations at admission, $n(\%)$} \\
\hline Palpable purpura & $33(100)$ & $28(90.3)$ & $30(96.8)$ & 0.148 \\
\hline Abdominal pain & $5(15.2)$ & $10(32.3)$ & $4(12.9)$ & 0.112 \\
\hline Arthritis & $6(18.2)$ & $4(12.9)$ & $3(9.7)$ & 0.613 \\
\hline Hypertension, $n(\%)$ & $7(21.2)$ & $9(29.0)$ & $7(22.6)$ & 0.756 \\
\hline MAP (mmHg) & $105 \pm 11$ & $111 \pm 14$ & $105 \pm 10$ & 0.244 \\
\hline White blood cell $\left(\times 10^{9} \mathrm{~L}^{-1}\right)$ & $8.2 \pm 2.4$ & $8.6 \pm 2.2$ & $8.9 \pm 2.3$ & 0.895 \\
\hline Hemoglobin $(\mathrm{g} / \mathrm{L})$ & $129 \pm 18$ & $132 \pm 14$ & $125 \pm 14$ & 0.252 \\
\hline Platelet $\left(\times 10^{9} \mathrm{~L}^{-1}\right)$ & $221 \pm 52$ & $238 \pm 70$ & $229 \pm 71$ & 0.559 \\
\hline Proteinuria (g/24 h) & $2.1 \pm 0.9^{*}$ & $1.9 \pm 1.0$ & $1.6 \pm 0.8$ & 0.118 \\
\hline Albumin $(\mathrm{g} / \mathrm{L})$ & $38.6 \pm 5.3$ & $39.8 \pm 5.6$ & $40.4 \pm 5.4$ & 0.416 \\
\hline $\mathrm{sCr}(\mu \mathrm{mol} / \mathrm{L})$ & $61.9 \pm 17.9$ & $62.7 \pm 20.3$ & $68.4 \pm 18.0$ & 0.330 \\
\hline eGFR $\left(\mathrm{ml} / \mathrm{min}\right.$ per $\left.1.73 \mathrm{~m}^{2}\right)$ & $118 \pm 33$ & $110 \pm 35$ & $108 \pm 28$ & 0.444 \\
\hline $\operatorname{IgA}(\mathrm{mg} / \mathrm{dl})$ & $277 \pm 66$ & $260 \pm 95$ & $307 \pm 111$ & 0.144 \\
\hline $\mathrm{CRP}(\mathrm{mmol} / \mathrm{L})$, median (range) & $1.8(1.0-88.5)$ & $2.1(1.0-56.7)$ & $2.2(1.0-52.8)$ & 0.867 \\
\hline $\operatorname{ESR}(\mathrm{mm} / \mathrm{h})$ & $13.9 \pm 10.3$ & $11.0 \pm 8.9$ & $15.6 \pm 13.9$ & 0.448 \\
\hline
\end{tabular}

Numerical data were expressed as mean \pm standard deviation. MAP: mean arterial pressure; sCr: serum creatine; eGFR: estimated glomerular filtration rate; IgA: immunoglobulin A; CRP: C reactive protein; ESR: erythrocyte sedimentation rate. ${ }^{*} P<0.05$ compared with the control group

Table 2 Pathological characteristics of patients in the MMF, CS, and control groups

\begin{tabular}{|c|c|c|c|c|}
\hline \multirow{2}{*}{ Characteristics } & \multicolumn{3}{|c|}{ Group } & \multirow{2}{*}{$P$-value } \\
\hline & MMF & $\mathrm{CS}$ & Control & \\
\hline Cases, $n$ & 33 & 31 & 31 & \\
\hline Pathological type, $n$ & & & & 0.156 \\
\hline II & 9 & 15 & 9 & \\
\hline IIIa & 16 & 11 & 19 & \\
\hline IIIb & 8 & 5 & 3 & \\
\hline \multicolumn{5}{|c|}{ Proportion of glomeruli with (\%), median (range) } \\
\hline Global sclerosis & $2.9(0-30.8)$ & $1.4(0-35.0)$ & $3.0(0-28.6)$ & 0.737 \\
\hline Crescent & $6.9(0-33.3)$ & $4.3(0-32.1)$ & $5.6(0-25.9)$ & 0.684 \\
\hline Mesangial proliferation, $n$ & & & & 0.184 \\
\hline Mild & 30 & 31 & 30 & \\
\hline Moderate & 3 & 0 & 1 & \\
\hline Glomerular segmental necrosis, $n$ & 2 & 2 & 0 & 0.489 \\
\hline Interstitial infiltrates, $n$ & & & & 0.173 \\
\hline Absent & 13 & 11 & 15 & \\
\hline Mild $(\leq 25 \%)$ & 18 & 15 & 16 & \\
\hline Moderate $(25 \%-50 \%)$ & 2 & 5 & 0 & \\
\hline
\end{tabular}

Numerical data were expressed as mean \pm standard deviation 

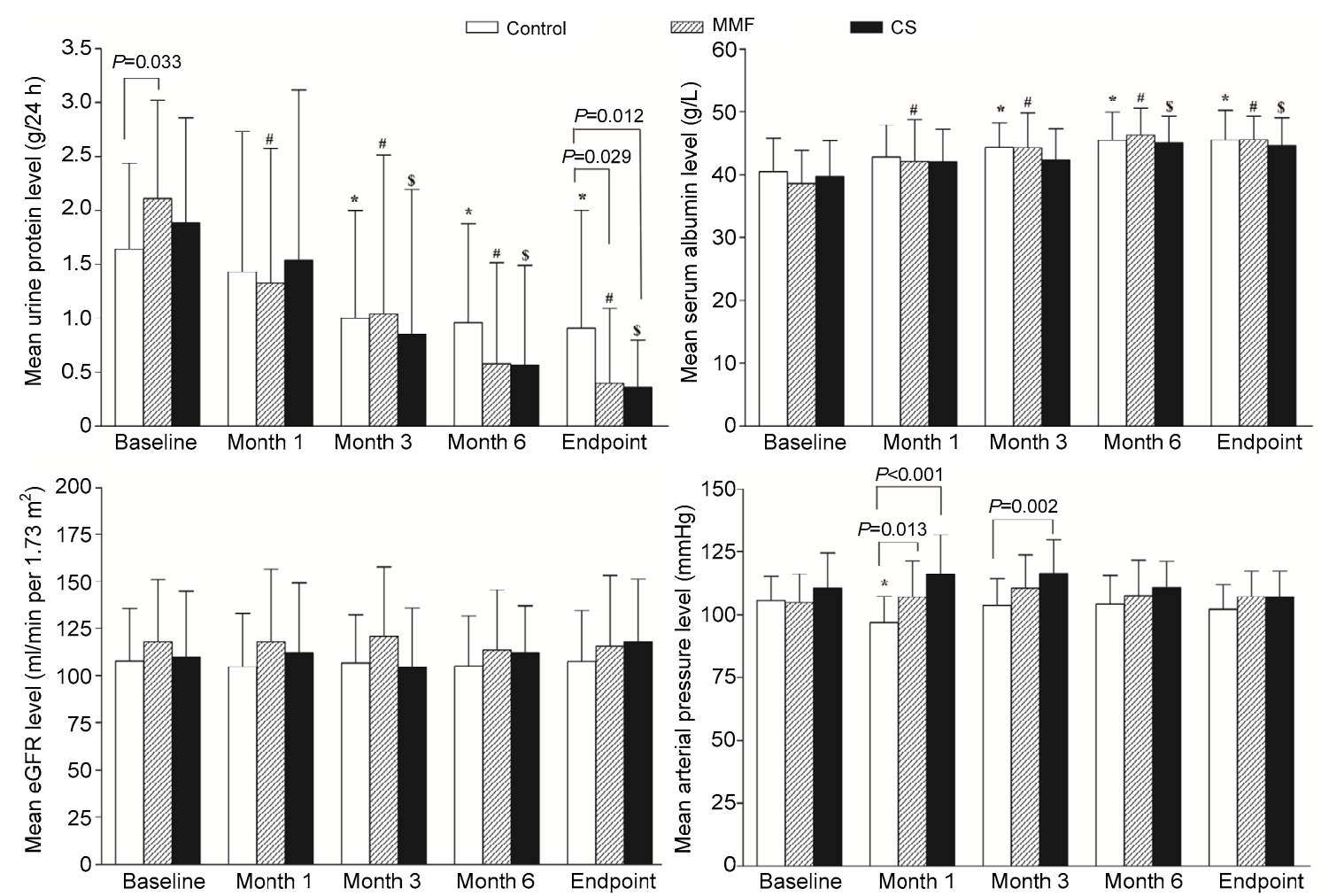

Fig. 1 Changes of urine protein, serum albumin, estimated glomerular filtration rate (eGFR), and mean arterial pressure (MAP) in the MMF, CS, and control groups during follow-up

${ }^{*} P<0.05$ compared with baseline in the control group; ${ }^{\#} P<0.05$ compared with baseline in the MMF group; ${ }^{\$} P<0.05$ compared with baseline in the CS group

at 6 months and $72.7 \%, 64.5 \%$, and $45.2 \%$ at the end of follow-up. The remission rate in the MMF group was significantly higher than that in the control group both at 6 months $(P=0.046)$ and at the end of follow-up $(P=0.025)$. There were 12 relapses including $2(6.1 \%)$ in the MMF group, $4(12.9 \%)$ in the CS group, and $6(19.4 \%)$ in the control group by the end of follow-up.

The overall number of reported adverse events was 17 in the MMF group, 30 in the CS group, and 6 in the control group $(P<0.001$; Table 3$)$. Thirteen patients (4 in the MMF group, 6 in the CS group, and 3 in the control group) experienced infections but no one was admitted to hospital. The patients in the CS group had a tendency for increased incidences of hyperlipidemia and hypertension compared with those in the MMF and control groups. One patient in the CS group had a bone fracture because of trauma after 8 months and then stopped prednisone with no relapse.

\section{Discussion}

In this study, we retrospectively analyzed the effects of MMF plus prednisone, full-dose prednisone, and ACEI or ARB therapy alone in HSPN patients with moderate proteinuria $(1.0-3.5 \mathrm{~g} / 24 \mathrm{~h})$. The HSPN patients in the MMF group had a higher remission rate both at 6 months and at the end of followup (median 28 months) compared with those in the control group, and they had a tendency for faster remission since their levels of proteinuria and serum albumin improved significantly after the first month. Compared with full-dose prednisone, MMF plus lowdose prednisone had the same remission rate, fewer adverse effects, and a tendency for fewer relapses at the end of follow-up.

HSPN makes up $0.6 \%-2.0 \%$ of nephritis in adults and less than that in children, and the incidence of progression to end stage renal disease is higher in adults than in children with a relative risk of 14.89 
Table 3 Adverse events in the MMF, CS, and control groups

\begin{tabular}{lcccc}
\hline \multirow{2}{*}{ Characteristics } & & Group & \multirow{2}{*}{$P$-value } \\
\cline { 2 - 4 } & MMF & CS & Control & $<0.001$ \\
Overall adverse events & $17^{* \#}$ & $30^{*}$ & 6 & 0.513 \\
Infection & 4 & 6 & 3 & 0.337 \\
$\quad$ Urinary tract infection & 0 & 2 & 1 & 0.740 \\
$\quad$ Bronchitis & 4 & 3 & 2 & 0.352 \\
$\quad$ Herpes zoster & 0 & 1 & 0 & 0.072 \\
Hyperlipidemia & 4 & $7^{*}$ & 1 & 0.152 \\
Hyperglycemia & 1 & 3 & 0 & 0.049 \\
Hypertension & 2 & $5^{*}$ & 0 & 0.352 \\
Insomnia & 0 & 1 & 0 & 0.352 \\
Alopecia & 0 & 1 & 0 & 0.348 \\
Osteoporosis & 1 & 2 & 0 & 0.352 \\
Bone fracture & 0 & 1 & 0 & 0.609 \\
Acne vulgaris & 1 & 1 & 0 & 0.824 \\
Gastrointestinal symptoms & 2 & 2 & 1 & 0.352 \\
Leukopenia & 1 & 0 & 0 & 0.609 \\
Alanine aminotransferase elevation & 1 & 1 & 0 & \\
\hline
\end{tabular}

Hyperlipidemia: blood cholesterol greater than $5.17 \mathrm{mmol} / \mathrm{L}$ and (or) triglycerides greater than $2.3 \mathrm{mmol} / \mathrm{L}$; Hyperglycemia: fasting plasma glucose greater than $6.1 \mathrm{mmol} / \mathrm{L}$; Hypertension: systolic blood pressure greater than $140 \mathrm{mmHg}$ and (or) diastolic blood pressure greater than $90 \mathrm{mmHg}$; Alanine aminotransferase elevation: serum alanine aminotransferase greater than $40 \mathrm{U} / \mathrm{L}$. ${ }^{*} P<0.05$ compared with the control group; ${ }^{\#} P<0.05$ compared with the CS group

( $P=0.014$ ) (Coppo et al., 2006). Pillebout et al. (2002) retrospectively studied a cohort of 250 adult HSP patients with a median of 14.8 years of follow-up. They found $11 \%$ of the patients reached end stage renal failure, $13 \%$ had severe renal failure $(\mathrm{CrCl}$ $<30 \mathrm{ml} / \mathrm{min}$ ), and $14 \%$ had moderate renal insufficiency $(\mathrm{CrCl}<50 \mathrm{ml} / \mathrm{min})$. As predictors of renal outcome, Coppo et al. (2006) revealed that the risk of progression of HSPN associated with increasing proteinuria level during follow-up was greater than the risks associated with decreased renal function, severe proteinuria, hypertension, or crescents present at onset. Therefore, persistent remission of proteinuria is important.

Inhibitors of the renin angiotensin system (RAS), such as ACEI or ARB, are the first choice for treatment of mild to moderate proteinuria. In this study, we included adult HSPN patients with proteinuria greater than $1.0 \mathrm{~g} / 24 \mathrm{~h}$ or less than $3.5 \mathrm{~g} / 24 \mathrm{~h}$ after at least three months of therapy of ACEI or ARB. Nearly half of the patients in the control group achieved remission after prolonged and enhanced (1.73-fold increase in dosage) inhibition of RAS. Therefore, in these patients, an enhanced dose may be suggested for ACEI or ARB therapy. Hypotension may be one concern. However, our results showed that there was a transient decrease in MAP level after the first month. Based on the vasculitic background, HSPN patients are commonly treated with CS (Oh et al., 2012). A systematic review, including three prospective, randomized, placebo-control trials and 12 retrospective trials, analyzed the efficacy of CS for HSP and found that early CS treatment at the onset of HSP significantly reduced the odds of developing persistent kidney disease (Weiss et al., 2007). However, these studies did not take account of the effects of CS on proteinuria or remission of HSPN and no ACEI or ARB was used as a control (Mollica et al., 1992; Saulsbury, 1993; Huber et al., 2004; Ronkainen et al., 2006). Based on the RCT data for IgAN, the KDIGO Glomerulonephritis Work Group (2012) suggested a six-month course of CS for HSPN patients with persistent proteinuria greater than $1 \mathrm{~g} / 24 \mathrm{~h}$, after a trial of ACEI or ARB. In this study, adding full-dose CS achieved a higher remission rate and a lower proteinuria level compared with the control, but the incidence of adverse effects was significantly higher, especially for hyperlipidemia and hypertension.

MMF has a strong immunosuppressive, antiproliferative effect, and fewer adverse effects on metabolism and fertility. Some HSP cases have been reported, in which patients received MMF treatment 
based on their vasculitic background and relatively young age (Nikibakhsh et al., 2010; Du et al., 2012). Nikibakhsh et al. (2010) used MMF (30 mg/kg) to treat six HSP children who were resistant to steroids, had steroid dependency or had obvious steroid side effects. All six patients achieved remission and did not relapse after 6-12 months of follow-up. Du et al. (2012) used MMF (20-25 mg/kg) to treat $12 \mathrm{HSPN}$ children who were resistant to steroid treatment and had an average proteinuria of $5.6 \mathrm{~g} / \mathrm{d}$. They found that after a mean of 2.5 months of MMF therapy all the children had responded, and that after 10-15 months of therapy, no child relapsed during 3.9 years of follow-up. In our previous study, we treated 27 adult HSPN patients with severe proteinuria with MMF combined with prednisone. After a median of 28.2 months, $77.8 \%$ of patients achieved remission, the same proportion as those receiving full-dose steroid therapy, and none relapsed while $19 \%$ of patients receiving full-dose steroid treatment relapsed (Ren et al., 2012). In this study, although the HSPN patients in the MMF group had more proteinuria at baseline, they had a higher remission rate compared with those in the control group and a tendency for faster remission. Compared with full-dose prednisone, MMF plus low-dose prednisone had the same remission rate, fewer adverse effects, and a tendency for fewer relapses at the end of follow-up.

This study had several limitations. First, it was a retrospective and non-randomized study, so doctors based treatment decisions on their own experiences and some baseline parameters were not balanced between groups. For example, the proteinuria level was higher in the MMF group than in the control group at baseline, indicating patients treated with MMF had more proteinuria, which may have resulted in a relatively low response to therapy. Second, the small number of cases sampled impaired the strength of the evidence. Third, the pathological characteristics of a severe HSPN were absent. Considering mild to moderate renal pathological changes and a good average eGFR level at baseline, the duration of follow-up was not sufficient to achieve endpoint events such as renal failure or death. So we need a prospective, well-controlled, and long-term study to verify the efficacy of MMF plus low-dose prednisone for treating adult HSPN patients.

\section{Conclusions}

MMF with low-dose prednisone may be as effective as full-dose prednisone and tends to have fewer adverse effects. Therefore, it is probably superior to conservative treatments of adult HSPN patients with moderate proteinuria.

\section{Compliance with ethics guidelines}

Fei HAN, Liang-liang CHEN, Ping-ping REN, Jing-yun LE, Pei-jing CHOONG, Hong-ju WANG, Ying XU, and Jiang-hua CHEN declare that they have no conflict of interest. All procedures followed were in accordance with the ethical standards of the responsible committee on human experimentation (institutional and national) and with the Helsinki Declaration of 1975, as revised in 2008 (5). Informed consent was obtained from all patients for being included in the study. Additional informed consent was obtained from all patients for whom identifying information is included in this article.

\section{References}

Chan, T.M., Li, F.K., Tang, C.S., et al., 2000. Efficacy of mycophenolate mofetil in patients with diffuse proliferative lupus nephritis. Hong Kong-Guangzhou Nephrology Study Group. N. Engl. J. Med., 343(16):1156-1162. [doi:10.1056/NEJM200010193431604]

Coppo, R., Mazzucco, G., Cagnoli, L., et al., 1997. Long-term prognosis of Henoch-Schönlein nephritis in adults and children. Italian Group of Renal Immunopathology Collaborative Study on Henoch-Schönlein purpura. Nephrol. Dial. Transplant., 12(11):2277-2283. [doi:10.1093/ndt/ 12.11.2277]

Coppo, R., Andrulli, S., Amore, A., et al., 2006. Predictors of outcome in Henoch-Schönlein nephritis in children and adults. Am. J. Kidney Dis., 47(6):993-1003. [doi:10. 1053/j.ajkd.2006.02.178]

Counahan, R., Winterborn, M.H., White, R.H., et al., 1977. Prognosis of Henoch-Schönlein nephritis in children. $B r$. Med. J., 2(6078):11-14. [doi:10.1136/bmj.2.6078.11]

Du, Y., Hou, L., Zhao, C., et al., 2012. Treatment of children with Henoch-Schönlein purpura nephritis with mycophenolate mofetil. Pediatr. Nephrol., 27(5):765-771. [doi:10.1007/s00467-011-2057-9]

Foster, B.J., Bernard, C., Drummond, K.N., et al., 2000. Effective therapy for severe Henoch-Schönlein purpura nephritis with prednisone and azathioprine: a clinical and histopathologic study. J. Pediatr., 136(3):370-375. [doi:10.1067/mpd.2000.103448]

Ginzler, E.M., Dooley, M.A., Aranow, C., et al., 2005. Mycophenolate mofetil or intravenous cyclophosphamide for lupus nephritis. N. Engl. J. Med., 353(21):2219-2228. [doi:10.1056/NEJMoa043731]

Han, F., Liu, G., Zhang, X., et al., 2011. Effects of mycophenolate 
mofetil combined with corticosteroids for induction therapy of microscopic polyangiitis. Am. J. Nephrol., 33(2):185-192. [doi:10.1159/000324364]

Hu, W., Liu, C., Xie, H., et al., 2008. Mycophenolate mofetil versus cyclophosphamide for inducing remission of ANCA vasculitis with moderate renal involvement. Nephrol. Dial. Transplant., 23(4):1307-1312. [doi:10. 1093/ndt/gfm780]

Huber, A.M., King, J., McLaine, P., et al., 2004. A randomized, placebo-controlled trial of prednisone in early Henoch Schonlein Purpura. BMC Med., 2(1):7. [doi:10.1186/ 1741-7015-2-7]

Kawasaki, Y., Suzuki, J., Suzuki, H., 2004. Efficacy of methylprednisolone and urokinase pulse therapy combined with or without cyclophosphamide in severe Henoch-Schöenlein nephritis: a clinical and histopathological study. Nephrol. Dial. Transplant., 19(4):858-864. [doi:10.1093/ndt/gfg617]

KDIGO (Kidney Disease: Improving Global Outcomes) Glomerulonephritis Work Group, 2012. KDIGO clinical practice guideline for glomerulonephritis. Kidney Int. Suppl., 2(2):139-274.

Kellerman, P.S., 2006. Henoch-Schönlein purpura in adults. Am. J. Kidney Dis., 48(6):1009-1016. [doi:10.1053/j.ajkd. 2006.08.031]

Mollica, F., Li, V.S., Garozzo, R., et al., 1992. Effectiveness of early prednisone treatment in preventing the development of nephropathy in anaphylactoid purpura. Eur. J. Pediatr., 151(2):140-144. [doi:10.1007/BF01958961]

Nikibakhsh, A.A., Mahmoodzadeh, H., Karamyyar, M., et al., 2010. Treatment of complicated Henoch-Schönlein purpura with mycophenolate mofetil: a retrospective case series report. Int. J. Rheumatol., 2010:254316. [doi:10. 1155/2010/254316]

Oh, H.J., Ahn, S.V., Yoo, D.E., et al., 2012. Clinical outcomes, when matched at presentation, do not vary between adult-onset Henoch-Schönlein purpura nephritis and IgA nephropathy. Kidney Int., 82(12):1304-1312. [doi:10. 1038/ki.2012.302]

Ozen, S., Ruperto, N., Dillon, M.J., et al., 2006. EULAR/PreS endorsed consensus criteria for the classification of childhood vasculitides. Ann. Rheum. Dis., 65(7):936-941. [doi:10.1136/ard.2005.046300]

Pillebout, E., Thervet, E., Hill, G., et al., 2002. HenochSchönlein Purpura in adults: outcome and prognostic factors. J. Am. Soc. Nephrol., 13(5):1271-1278. [doi:10. 1097/01.ASN.0000013883.99976.22]

Pillebout, E., Alberti, C., Guillevin, L., et al., 2010. Addition of cyclophosphamide to steroids provides no benefit compared with steroids alone in treating adult patients with severe Henoch Schonlein Purpura. Kidney Int., 78(5):495-502. [doi:10.1038/ki.2010.150]

Ren, P., Han, F., Chen, L., et al., 2012. The combination of mycophenolate mofetil with corticosteroids induces remission of Henoch-Schönlein purpura nephritis. Am. $J$. Nephrol., 36(3):271-277. [doi:10.1159/000341914]

Ronkainen, J., Nuutinen, M., Koskimies, O., 2002. The adult kidney 24 years after childhood Henoch-Schönlein purpura: a retrospective cohort study. Lancet, 360(9334): 666-670. [doi:10.1016/S0140-6736(02)09835-5]

Ronkainen, J., Koskimies, O., Ala-Houhala, M., et al., 2006. Early prednisone therapy in Henoch-Schönlein purpura: a randomized, double-blind, placebo-controlled trial. $\mathrm{J}$. $\mathrm{Pe}$ diatr., 149(2):241-247. [doi:10.1016/j.jpeds.2006.03.024]

Saulsbury, F.T., 1993. Corticosteroid therapy does not prevent nephritis in Henoch-Schönlein purpura. Pediatr. Nephrol., 7(1):69-71. [doi:10.1007/BF00861574]

Shenoy, M., Bradbury, M.G., Lewis, M.A., et al., 2007. Outcome of Henoch-Schönlein purpura nephritis treated with long-term immunosuppression. Pediatr. Nephrol., 22(10): 1717-1722. [doi:10.1007/s00467-007-0557-4]

Tang, S.C., Tang, A.W., Wong, S.S., et al., 2010. Long-term study of mycophenolate mofetil treatment in IgA nephropathy. Kidney Int., 77:543-759. [doi:10.1038/ki. 2009.499]

Weiss, P.F., Feinstein, J.A., Luan, X., et al., 2007. Effects of corticosteroid on Henoch-Schönlein purpura: a systematic review. Pediatrics, 120(5):1079-1087. [doi:10.1542/ peds.2007-0667]

\section{中文概要}

题 目: 霉酚酸酯联合糖皮质激素诱导治疗过敏性紫癜 性肾炎的回顾性研究

目 的: 合并中等量蛋白尿的过敏性紫癜性肾炎的诱导治 疗尚无确切方案, 本研究通过回顾性比较分析了 霉酚酸酯联合糖皮质激素的疗效与安全性。

创新点: 首次对霉酚酸酯联合糖皮质激素诱导治疗过敏性 紫癜性肾炎进行了回顾性研究。

方 法: 回顾性分析 2007 年 1 月至 2013 年 6 月间在浙江 大学附属第一医院肾脏病中心接受肾穿刺活检,

且经过 3 个月以上血管紧张素转换酶抑制剂 (ACEI) /血管紧张素受体拮抗剂 (ARB) 治疗 后蛋白尿为 1.0 3.5 g/24 h 的过敏性紫癜性肾炎 患者 95 例。根据治疗方案分为 3 组, 霉酚酸酯 组（33 例）在原剂量 $\mathrm{ACEI} / \mathrm{ARB}$ 的基础上加用 霉酚酸酯联合低剂量糖皮质激素, 糖皮质激素组 (31 例) 在原剂量 $\mathrm{ACEI} / \mathrm{ARB}$ 的基础上加用全 剂量糖皮质激素, 对照组（31 例）维持单用 ACEI/ARB 治疗, 但可提高其剂量。患者随访观 察 6 78 月 (中位观察时间 28 月), 霉酚酸酯组、 糖皮质激素组与对照组的蛋白尿缓解率分别为 $72.7 \% 、 64.5 \%$ 与 $45.2 \%$ （图 1)，发生副作用分 别为 17 例、 30 例与 6 例, 糖皮质激素组高脂血 症与高血压发生率较高（表 3）。

结 论: 霉酚酸酯联合低剂量糖皮质激素可有效诱导缓解 过敏性紫癜性肾炎, 其缓解率与全剂量糖皮质激 素治疗相当, 且副作用较少。

关键词：过敏性紫癜性肾炎；霉酚酸酯；缓解率 